\title{
A NOTE ON COMPLETE INTERSECTIONS
}

BY

S. M. BHATWADEKAR

\begin{abstract}
Let $R$ be a regular local ring and let $R[T]$ be a polynomial algebra in one variable over $R$. In this paper the author proves that every maximal ideal of $R[T]$ is complete intersection in each of the following cases: (1) $R$ is a local ring of an affine algebra over an infinite perfect field, (2) $R$ is a power series ring over a field.
\end{abstract}

Introduction. Let $R$ be a regular local ring. Let $R[T]$ be a polynomial algebra in one variable over $R$. In [D-G] the following question has been asked.

Question. Is every maximal ideal of $R[T]$ complete intersection?

In this paper we prove that the answer to the above question is affirmative in each of the following cases:

(1) $R$ is a local ring of an affine algebra over an infinite perfect field.

(2) $R$ is a power series ring over a field.

This paper is divided into three sections. In $\S 1$ we fix notations and state a theorem without proof which is used in $\$ \S 2$ and 3. In $\$ 2$ we prove some lemmas and propositions which are used in proving the result when $R$ is a local ring of an affine algebra. $\$ 3$ deals with the power series case.

1. Throughout this paper we consider commutative noetherian rings with 1 . For a ring $R, \operatorname{dim} R$ denotes its Krull dimension which we always assume to be finite. If $R$ is a local ring then $\Re(R)$ will always denote its unique maximal ideal. If $M$ is a finitely generated $R$-module then $\mu(M)$ will denote the minimal number of generators of $M$. For an ideal $I$ of $R \mathrm{ht}(I)$ denotes the height of $I$.

Definition. Let $I$ be an unmixed ideal of $R$ of height $r$. Then $I$ is said to be complete intersection in $R$ if $I=\sum_{i=1}^{r} R a_{i}$, where $a_{1}, a_{2}, \ldots, a_{r}$ is a regular $R$ sequence.

REMARK. If $R$ is Cohen-Macaulay then $I$ is complete intersection if and only if $\mu(I)=\mathrm{ht}(I)$.

Let $R$ and $S$ be two local rings.

Definition. $R$ is said to be a local extension of $S$ if $S$ is a subring of $R$ and $\Re(S)=\Re(R) \cap S . R$ is said to be unramified over $S$ if $\Re(S) R=\Re(R)$ and $R / \mathfrak{R}(R)$ is separable over $S / \mathfrak{T}(S)$.

Let $L / K$ be a finite separable extension of $K$. Then $L$ is a simple extension of $K$. By a minimal polynomial of $L$ over $K$ we always mean an irreducible monic polynomial over $K$ satisfied by a generator of $L$ over $K$.

Now we state a theorem which has been proved in [D-G, Theorem 3].

Received by the editors November 26, 1980.

1980 Mathematics Subject Classification. Primary 13B25; Secondary 13F20.

(C) 1982 American Mathematical Society 0002-9947/81/0000-1029/\$02.75 
THEOREM. Let $R$ be a regular ring. Let $A=R[X, Y]$ be a polynomial algebra in two variables over $R$. Then every maximal ideal of $A$ is complete intersection.

In subsequent sections this theorem will always be referred to as the D-G theorem.

2. In this section we prove the following theorem.

THEOREM 2.1. Let $k$ be an infinite perfect field. Let $C$ be an affine $k$-algebra. Let $\mathfrak{\Im}$ be a prime ideal of $C$ such that $C_{\Im}=R$ is regular. Let $M$ be a maximal ideal of $R[T]$. Then $M$ is complete intersection.

For the proof of this theorem we need some lemmas and propositions.

LEMмa 2.2. Let $A$ be an affine domain of $\operatorname{dim} 1$ over a field $K$. Let $\bar{\Re}$ be a nonregular maximal ideal of $A$ such that $A \overline{/ \mathfrak{T}}$ is a finite separable (therefore simple) extension of $K$. Then there exist $y_{1}, y_{2}, \ldots, y_{r} \in A$ such that

(1) $A$ is integral over $K\left[y_{1}\right]$,

(2) the inclusion map $K\left[y_{1}\right] / \overline{\mathscr{M}} \cap K\left[y_{1}\right] \rightarrow A / \overline{\mathscr{M}}$ is an isomorphism,

(3) $\overline{\mathscr{M}}=\left(f\left(y_{1}\right), y_{2}, \ldots, y_{r}\right)$ where $r=\mu\left(\overline{\mathscr{M}} / \overline{\mathscr{M}}^{2}\right)$ and $f$ is a minimal polynomial of $A / \overline{\text { TR }}$ over $K$.

Proof. Since $A$ is one dimensional and $\bar{\Re}$ nonregular we have $\mu\left(\overline{\mathscr{M}} / \overline{\mathscr{M}}^{2}\right)=r \geqslant$ $2=\operatorname{dim} A+1$. Therefore by [Mo, Corollary 3] it follows that $\mu(\overline{\mathfrak{M}})=\mu\left(\overline{\mathfrak{M}} / \overline{\mathfrak{M}}^{2}\right)$.

Let $A / \overline{O R}=K[\alpha]$. Let $f(X)$ be the minimal polynomial of $\alpha$ over $K$. Let $b \in A$ be such that $\alpha=b \bmod \bar{\Re}$. Then $\alpha$ is separable over $K$ and $f(X)$ is its minimal polynomial imply that $f(b) \in \bar{\Re}$ and $\partial f(b) / \partial X \notin \bar{\Re}$. If $f(b) \in \bar{\Re}^{2}$ then replacing $b$ by $b+x$ for some $x \in \overline{\mathscr{M}}-\overline{\mathscr{M}}^{2}$ we get $f(b) \notin \overline{\mathfrak{M}^{2}}$. This in particular implies that $b$ is not algebraic over $K$.

Since $A$ is one dimensional affine, by the normalization theorem [Z-S, p. 200] there exists $y \in A$ such that $A$ is integral over $K[y]$. Let $\bar{\Re} \cap K[y]=(h(y))$. Let $y_{1}=b+h(y)^{l}$ where $l$ is a positive integer. Then by taking sufficiently large $l \geqslant 2$ one can see that $K[y, b]=K\left[y_{1}, y\right]$ is integral over $K\left[y_{1}\right]$. Moreover

$$
f\left(y_{1}\right)=f(b)+(\partial f / \partial X)(b) h(y)^{l}+c h(y)^{2 l}, \quad c \in K[y, b] .
$$

Since $f(b) \notin \overline{\mathscr{M}}^{2}, h(y) \in \bar{\Re}$ and $l \geqslant 2$ we get $f\left(y_{1}\right) \in \overline{\mathfrak{M}}-\overline{\mathscr{M}}^{2}$. Since $A$ is integral over $K[y, b], A$ is integral over $K\left[y_{1}\right]$ and $\overline{\mathscr{N}} \cap K\left[y_{1}\right]=\left(f\left(y_{1}\right)\right)$. Therefore the inclusion map $K\left[y_{1}\right] / \overline{\mathscr{R}} \cap K\left[y_{1}\right] \rightarrow A / \bar{\Re}$ is an isomorphism.

Let $A^{\prime}=A /\left(f\left(y_{1}\right)\right)$, $\mathfrak{K}^{\prime}=\overline{\mathscr{N}} /\left(f\left(y_{1}\right)\right)$. Then $A^{\prime}$ is zero dimensional and $\mu\left(\mathfrak{M}^{\prime} / \mathcal{M}^{\prime 2}\right)=\mu\left(\overline{\mathscr{N}} / \overline{\mathfrak{M}}^{2}\right)-1=r-1 \geqslant 1$. Therefore by [Mo, Corollary 3] there exist $y_{2}^{\prime}, y_{3}^{\prime}, \ldots, y_{r}^{\prime} \in A^{\prime}$ such that $\Re^{\prime}=\left(y_{2}^{\prime}, y_{3}^{\prime}, \ldots, y_{r}^{\prime}\right)$. Let $y_{i}$ be a pull back of $y_{i}^{\prime}$ in $A$ for $2 \leqslant i \leqslant r$. Then $\bar{\Re}=\left(f\left(y_{1}\right), y_{2}, y_{3}, \ldots, y_{r}\right)$.

This completes the proof of Lemma 2.2. Now we state a lemma the proof of which is easy and can be found in [L, Lemma 2].

LeMma 2.3. Let $k$ be a perfect field. Let $C$ be an affine $k$-algebra. Let $\mathfrak{\Im}$ be a prime ideal of $C$ such that $C_{\Im}=R$ is regular. Then there exists a field extension $K / k$ and regular affine $K$-domain $B$ contained in $R$ such that

(1) $R=B_{\mathfrak{N}}$ for some maximal ideal M of $B$,

(2) $B / \mathfrak{R}=R / \mathscr{T}(R)$ is a finite separable extension of $K$. 
The following two propositions are very crucial for the proof of Theorem 2.1.

Proposition 2.4. Let $k, C, \mathfrak{I}, R, K, B$, N be as in Lemma 2.3. Let $p$ be a prime ideal of $R$ such that $R / p$ is one dimensional and nonregular. Then $R$ contains a local domain $S$ such that

(1) $S$ is a localization of a polynomial algebra $C^{\prime}$ over $K$ at some maximal ideal $\eta$ of $C^{\prime}$,

(2) there exists $h \in p \cap S$ such that the inclusion of $S$ in $R$ gives rise to an inclusion of $S / h S$ in $R / h R$ which is an isomorphism, i.e. $S / h S=R / h R$.

Proof. Since $R=B_{\mathfrak{R}}$ there exists a prime ideal $q$ of $B$ such that $q B_{\Re}=p$. Then $B / q$ is one dimensional and $\Re / q$ is a nonregular maximal ideal of $B / q$.

Let $A=B / q, \bar{\Re}=\Re / q$. Then by Lemma 2.2 there exist $y_{1}, y_{2}, \ldots, y_{r} \in A$ satisfying properties 1,2 and 3 of Lemma 2.2. Let $\phi: B \rightarrow A(=B / q)$ be the canonical map. Let $x_{i} \in B$ be such that $\phi\left(x_{i}\right)=y_{i}$ for $1 \leqslant i \leqslant r$. Then $q+$ $\left(f\left(x_{1}\right), x_{2}, \ldots, x_{r}\right)=\mathfrak{R}$ and $f\left(x_{1}\right), x_{2}, \ldots, x_{r}$ generate $\mathfrak{K} \bmod \mathfrak{M}^{2}+q$ where $r=$ $\operatorname{dim}_{B / \mathfrak{N}}\left(\mathfrak{N} / \mathfrak{N}^{2}+q\right)$. Let $\operatorname{dim}_{B / \mathfrak{N}}\left(\mathfrak{N} / \mathfrak{N}^{2}\right)=\mu\left(\mathfrak{N} B_{\mathfrak{N}}\right)=\operatorname{dim} R=n$. Then since we have the following exact sequence

$$
0 \rightarrow q / q \cap \mathfrak{M}^{2} \rightarrow \mathfrak{M} / \Re^{2} \rightarrow \mathfrak{K} / \mathscr{N}^{2}+q \rightarrow 0
$$

we get $\operatorname{dim}_{B / \Re}\left(q / q \cap \Re^{2}\right)=n-r$. Let $x_{r+1}, x_{r+2}, \ldots, x_{n} \in q$ be such that $\left(x_{r+1}, x_{r+2}, \ldots, x_{n}\right)+q \cap \mathfrak{M}^{2}=q$. Then it is easy to see that $\left(f\left(x_{1}\right), \ldots, x_{r}, x_{r+1}, \ldots, x_{n}\right)+\mathfrak{N}^{2}=\mathfrak{T}$. Since $R=B_{\mathfrak{R}}$ is regular of $\operatorname{dim} n$ it follows that $\left(f\left(x_{1}\right), x_{2}, \ldots, x_{n}\right) R=\mathscr{T}(R)$ and $f\left(x_{1}\right), x_{2}, \ldots, x_{n}$ are algebraically independent over $K$. Therefore $x_{1}, x_{2}, \ldots, x_{n}$ are also algebraically independent over $K$ and hence $C^{\prime}=K\left[x_{1}, x_{2}, \ldots, x_{n}\right]$ is a polynomial algebra over $K$ contained in $B$.

Let $\eta=C^{\prime} \cap \mathfrak{T}$. Then $\eta=\left(f\left(x_{1}\right), x_{2}, \ldots, x_{n}\right)$ is a maximal ideal of $C^{\prime}$ and the inclusion map $C^{\prime} / \eta \rightarrow B / \mathscr{T}$ is an isomorphism. Moreover $A(=B / q)$ is integral over $C^{\prime} / q_{1}$ where $q_{1}=q \cap C^{\prime}$ and $\overline{\mathfrak{T}}$ is the only maximal ideal of $A$ lying over the maximal ideal $\eta / q_{1}$ of $C^{\prime} / q_{1}$.

Let $L=$ quotient field of $B, L^{\prime}=$ quotient field of $C^{\prime}$. Then since $B$ and $C^{\prime}$ are affine $K$-domains of $\operatorname{dim} n, L$ is a finite algebraic extension of $L^{\prime}$. Let $B^{\prime}$ be the integral closure of $C^{\prime}$ in $L$. Then $B^{\prime}$ is a finitely generated $C^{\prime}$-module contained in $B$.

Let $\mathfrak{K}^{\prime}=\mathfrak{T} \cap B^{\prime}, B_{\mathfrak{N}}^{\prime}=R^{\prime}, C_{\eta}^{\prime}=S$. Then we get a tower of local extensions $S \hookrightarrow R^{\prime} \hookrightarrow R$. Since $S / \mathfrak{M}(S)=C^{\prime} / \eta \stackrel{\sim}{\rightarrow} B / \mathfrak{N}=R / \mathscr{N}(R)$ and $R$ is unramified over $S, R$ is also unramified over $R^{\prime}$ and $R^{\prime} / \mathscr{N}\left(R^{\prime}\right) \stackrel{\sim}{\rightarrow} R / \mathscr{N}(R)$. But since $R^{\prime}$ and $R$ have the same quotient field $L$ and $R^{\prime}$ is normal, by Zariski's main theorem [BI, p. 93] we have $R^{\prime}=R$.

Let $q^{\prime}=q \cap B^{\prime}$. Then we get a tower of integral extensions $C^{\prime} / q_{1} \hookrightarrow B^{\prime} / q^{\prime} \hookrightarrow B / q$ $(=A)$. Since $\bar{\Re}(=\Re / q)$ is the only maximal ideal of $A$ lying over $\eta / q_{1}, \Re^{\prime} / q^{\prime}$ will be the only maximal ideal of $B^{\prime} / q^{\prime}$ lying over $\eta / q_{1}$. Therefore $\eta B^{\prime}+q^{\prime}$ is $\Re^{\prime}$-primary. Since $B_{\mathscr{N}}^{\prime}=R^{\prime}=R$ and $\eta R=\mathscr{R}(R)$ we have $\eta B^{\prime}+\Re^{\prime 2}=\Re^{\prime}$. But this implies that $\eta B^{\prime}+\mathfrak{N}^{\prime \prime}=\mathfrak{N}^{\prime}$ for every positive integer $l$. Since $\eta B^{\prime}+q^{\prime}$ is $\Re^{\prime}$-primary, there exists a positive integer, say $l_{0}$, such that $\Re^{\prime l_{0}} \subset \eta B^{\prime}+q^{\prime}$. Therefore $\eta B^{\prime}+q^{\prime}=\mathscr{N}^{\prime}$. Moreover $\eta B^{\prime}+\mathfrak{K}^{\prime 2}=\mathfrak{M}^{\prime}$ implies that $\mathscr{N}{ }^{\prime} / \eta B^{\prime}$ is an 
idempotent and therefore principal ideal of $B^{\prime} / \eta B^{\prime}$. Hence there exists $t \in q^{\prime}$ such that $t B^{\prime}+\eta B^{\prime}=\Re{ }^{\prime}$.

Let $B^{\prime \prime}=C^{\prime}[t], \mathfrak{T}^{\prime \prime}=\mathfrak{T}^{\prime} \cap B^{\prime \prime}, q^{\prime \prime}=q^{\prime} \cap B^{\prime \prime}$. It is obvious that $\mathfrak{R}^{\prime \prime} B^{\prime}=\mathfrak{R}^{\prime}$ and $B^{\prime \prime} / \mathscr{N} " \stackrel{\sim}{\rightarrow} B^{\prime} / \Re^{\prime}$. Since $B^{\prime}$ is a finitely generated $B^{\prime \prime}$-module we have $B_{\Re}^{\prime \prime}=$ $B_{\Re^{\prime}}^{\prime}=R$ and $q^{\prime \prime} R=p$.

Since $B^{\prime \prime}$ is a simple integral extension of $C^{\prime}$ and $C^{\prime}$ is a unique factorization domain we get $B^{\prime \prime}=C^{\prime}[T] /(g(T))$ where $g(T)$ is a monic irreducible polynomial in $T$.

Let $\psi: C^{\prime}[T] \rightarrow B^{\prime \prime}\left(=C^{\prime}[t]\right)$ be the canonical map. Let $M=\psi^{-1}\left(\mathscr{T}{ }^{\prime \prime}\right)$. Since $\psi(T)=t \in \Re^{\prime \prime}$ we have $T \in M$. Also $\Re^{\prime \prime} \cap C^{\prime}=\eta$ implies $M \cap C^{\prime}=\eta$. Therefore $M=T C^{\prime}[T]+\eta C^{\prime}[T]$.

Let $g(T)=T^{i}+a_{i-1} T^{i-1}+\cdots+a_{1} T+a_{0}$. Then $g(t)=0$ and $t \in q^{\prime \prime}$ implies $a_{0} \in q_{1}=q^{\prime \prime} \cap C^{\prime}$. Since $B_{\mathscr{N}}^{\prime \prime \prime}=R$ and $\eta R=\Re(R)$ it follows that $a_{1} \notin \eta$, and therefore $t R=h R$ where $h=a_{0}$. Therefore the map $S / h S \rightarrow R / h R$ is an isomorphism. Thus the proof of Proposition 2.4 is complete.

Remark. Under the assumptions of Proposition 2.4 Lindel [L, Proposition 2] also has shown the existence of $S$ and $h$. Our proof is a variation of his proof because of the requirement that $h$ should belong to $p$.

Proposition 2.5. Let $K$ be an infinite field. Let $D=K\left[X_{1}, X_{2}, \ldots, X_{n}\right]$ be a polynomial algebra over $K$. Let $\Re=\left(f\left(X_{1}\right), X_{2}, \ldots, X_{n}\right)$ be a maximal ideal of $D$. Let $p$ be a prime ideal of $\operatorname{dim} 1$ contained in $\Re$. If $n \geqslant 3$ then $D$ contains a K-algebra $D^{\prime}$ of $\operatorname{dim} n-1$ such that

(1) $D=D^{\prime}[Y]$,

(2) $p+\Re^{\prime} D$ is $\Re$-primary where $\Re^{\prime}=\mathfrak{N} \cap D^{\prime}$.

Proof. If $p$ contains one of the generators $f\left(X_{1}\right), X_{2}, \ldots, X_{n}$, say $f\left(X_{1}\right)$, then $p+\left(X_{2}, \ldots, X_{n}\right)=\Re$. Therefore by taking $D^{\prime}=K\left[X_{2}, \ldots, X_{n}\right]$ we get the required result.

Now we assume that $X_{i} \notin p$ for $2 \leqslant i \leqslant n$ and $f\left(X_{1}\right) \notin p$. Then $p+\left(X_{n}\right)=I$ is a zero dimensional ideal of $D$ and hence contained in only finitely many maximal ideals of $D$. Let $T=\left\{\Re_{R}=\Re_{1}, \Re_{2}, \ldots, \Re_{t}\right\}$ be a finite set of maximal ideals of $D$ containing $I$.

For every $i, 2 \leqslant i \leqslant t$, let $V_{i}$ denote a subspace of $K^{n}$ consisting of $n$-tuples $\left(\lambda_{1}, \ldots, \lambda_{n}\right)$ such that $\lambda_{1} f\left(X_{1}\right)+\lambda_{2} X_{2}+\cdots+\lambda_{n} X_{n} \in \Re_{i}$. Then $V_{i} \neq K^{n}$ for $2 \leqslant i$ $\leqslant t$. Since $K$ is infinite we have $\cup_{2 \leqslant i \leqslant t} V_{i} \neq K^{n}$. Let $\left(\beta_{1}, \beta_{2}, \ldots, \beta_{n}\right)$ be such that $\left(\beta_{1}, \beta_{2}, \ldots, \beta_{n}\right) \notin V_{i}$ for every $i, 2 \leqslant i \leqslant t$. Let $Z=\beta_{1} f\left(X_{1}\right)+\beta_{2} X_{2}+\cdots+\beta_{n} X_{n}$. Since $X_{n} \in \mathfrak{M}_{i}$ for every $i, 2 \leqslant i \leqslant t$, we have $\beta_{l} \neq 0$ for some $l, 1 \leqslant l \leqslant n-1$.

If $\beta_{2}=0$ then taking $D^{\prime}=D\left[X_{1}, X_{3}, \ldots, X_{n}\right]$ we get $X_{n}, Z \in \mathfrak{N}^{\prime}=\mathfrak{N} \cap D^{\prime}$, and the ideal $p+\left(X_{n}, Z\right) D$ is $\Re$-primary. Therefore $p+\Re^{\prime} D$ is $\Re$-primary. Since $D=D^{\prime}\left[X_{2}\right]$ we get the required result.

If $\beta_{2} \neq 0$ then obviously $D=K\left[X_{1}, Z, X_{3}, \ldots, X_{n}\right]$. Taking $D^{\prime}=K\left[Z, X_{3}, \ldots, X_{n}\right]$ we get $X_{n}, Z \in \mathfrak{M}^{\prime}=\mathfrak{\pi} \cap D^{\prime}$. Therefore as before we see that $\mathfrak{K}^{\prime} D+p$ is N-primary. Since $D=D^{\prime}\left[X_{1}\right]$ we get the required result. 
Proof of Theorem 2.1. Let $p=M \cap R$. Then $\operatorname{dim} R / p \leqslant 1$. If $R / p$ is regular then since $\operatorname{ht}(M / p R[T])=1, M / p R[T]$ is a principal ideal of $R / p[T]$. Therefore

$$
\mu(M) \leqslant 1+\mu(p R[T])=1+\mu(p)=1+\mathrm{ht}(p)=\mathrm{ht}(M) .
$$

Since we always have $\operatorname{ht}(M) \leqslant \mu(M)$ we get the equality $\mu(M)=\operatorname{ht}(M)$ which shows that $M$ is complete intersection.

Now we suppose that $R / p$ is not regular. Then $\operatorname{dim} R / p=1, \operatorname{ht}(M)=\operatorname{ht}(p)+1$ $=\operatorname{dim} R$ and $\operatorname{dim} R \geqslant 2$.

Case $1 . \operatorname{dim} R=2$. Then $\operatorname{dim} R / p=1 \mathrm{implies} \operatorname{ht}(p)=1$. Therefore we have $\operatorname{ht}(M)=\operatorname{ht}(p)+1=2$. Since $R[T]$ is regular, $M$ is locally generated by a regular sequence of length 2 . Therefore $\operatorname{hd}_{R[T]} M=1$ where hd ${ }_{R[T]} M$ denotes the homological dimension of the $R[T]$-module $M$. Since

$$
\operatorname{Ext}_{R[T]}^{1}(M, R[T]) \simeq \operatorname{Ext}_{R[T]}^{2}(R[T] / M, R[T]) \simeq R[T] / M,
$$

we get $\operatorname{Ext}_{R[T]}^{1}(M, R[T])$ to be a cyclic $R[T]$-module. Therefore by [S, p. 8] there is an exact sequence $0 \rightarrow R[T] \rightarrow P \rightarrow M \rightarrow 0$ with $P$ finitely generated projective $R[T]$-module of rank 2. But by [Mu, Theorem] $P$ is free. Therefore $\mu(P)=2$. Since $M$ is an epimorphic image of $P$ we have

$$
\mu(M) \leqslant \mu(P)=2=\operatorname{ht}(M) \leqslant \mu(M) .
$$

Hence $M$ is complete intersection.

Case 2. $\operatorname{dim} R=n \geqslant 3$. By Lemma 2.3 and Proposition 2.4 there exist a field extension $K / k$ and a local domain $S$ contained in $R$ such that

(1) $S=K\left[X_{1}, \ldots, X_{n}\right]_{\eta}$ where $\eta$ is a maximal ideal of $K\left[X_{1}, \ldots, X_{n}\right]$ generated by $f\left(X_{1}\right), X_{2}, \ldots, X_{n}$ for some irreducible monic polynomial $f(X)$ over $K$.

(2) There exists $h \in p \cap S$ such that $S / h S=R / h R$ and therefore $S[T] / h S[T]=$ $R[T] / h R[T]$.

Let $\tilde{M}=M \cap S[T]$. Since $h \in \tilde{M}, \tilde{M}$ is a maximal ideal of $S[T]$. Moreover $\tilde{M} R[T]=M$ and $\operatorname{ht}(\tilde{M})=\operatorname{ht}(M)$. Therefore it is enough to prove that $\tilde{M}$ is a complete intersection ideal of $S[T]$.

Let $q=S \cap p=\tilde{M} \cap S$. Then $h \in q$ and hence $S / q=R / p$. Therefore $\operatorname{dim} S / q$ $=1$. Let $D=K\left[X_{1}, \ldots, X_{n}\right], \tilde{M}^{\prime}=\tilde{M} \cap D[T], q^{\prime}=q \cap D=\tilde{M}^{\prime} \cap D$. Then since $D_{\eta}=S$ we have $\tilde{M}^{\prime} S[T]=\tilde{M}, \operatorname{ht}\left(\tilde{M}^{\prime}\right)=\operatorname{ht}(\tilde{M})=n=\operatorname{dim} D$ and ht $\left(q^{\prime}\right)=\operatorname{ht}(q)=$ $n-1$. Therefore $\operatorname{dim} q^{\prime}=\operatorname{dim} D / q^{\prime}=1$.

Since $n \geqslant 3$ by Proposition 2.5 there exists a subalgebra $D^{\prime}$ of $D$ of $\operatorname{dim} n-1$ such that

(1) $D=D^{\prime}[Y]$,

(2) $\eta^{\prime} D+q^{\prime}$ is $\eta$-primary where $\eta^{\prime}=\eta \cap D^{\prime}$.

Consider the following commutative diagram

$$
\begin{array}{rrrr}
D^{\prime} \quad \hookrightarrow \quad & D^{\prime}[Y]=D & \hookrightarrow & D_{\eta}=S \\
& & \eta \\
& D[T] \hookrightarrow . & S[T]
\end{array}
$$

$\tilde{M}^{\prime}$ is a prime ideal of $D[T]$ of height $n=\operatorname{dim} D[T]-1$. Therefore every prime ideal of $D[T]$ which contains $\tilde{M}^{\prime}$ properly is a maximal ideal of $D[T]$. Let $M_{1}$ be one 
such maximal ideal. Then since $D^{\prime}, D, D[T]$ all are affine rings, $N_{1}=M_{1} \cap D^{\prime}$ will be a maximal ideal of $D^{\prime}$. If $\eta^{\prime}=N_{1}$ then since $\tilde{M}^{\prime} \subset M_{1}$ we have $\eta^{\prime} D+q^{\prime} \subset M_{1} \cap$ $D$. But $\eta^{\prime} D+q^{\prime}$ is $\eta$-primary and $\eta$ is maximal; therefore $\eta=M_{1} \cap D$. Since $S=D_{\eta}, \eta=M_{1} \cap D$ implies that $M_{1} S[T]$ is a prime ideal of $S[T]$ which contains $\tilde{M}^{\prime} S[T]=\tilde{M}$ properly which contradicts the fact that $\tilde{M}$ is maximal. Therefore $N_{1} \neq \eta^{\prime}$.

The above discussion shows that no prime ideal of $D[T]$ which contains $\tilde{M}^{\prime}$ properly can lie over a prime ideal of $D^{\prime}$ contained in $\eta^{\prime}$. Therefore $\tilde{M}^{\prime} S^{\prime}[T]$ becomes a maximal of $S^{\prime}[T]$ of height $=h t\left(\tilde{M}^{\prime}\right)$ where $S^{\prime}=D_{\eta^{\prime}}^{\prime}[Y]$. Then by the D-G theorem $\tilde{M}^{\prime} S^{\prime}[T]$ is complete intersection. Now we have the following tower of rings:

$$
D^{\prime}[Y, T]=D[T] \hookrightarrow S^{\prime}[T] \hookrightarrow S[T] .
$$

Since $\tilde{M}^{\prime} S^{\prime}[T]$ is complete intersection, $\tilde{M}^{\prime} S[T]=\tilde{M}$ and $\operatorname{ht}\left(\tilde{M}^{\prime} S^{\prime}[T]\right)=\operatorname{ht}\left(\tilde{M}^{\prime}\right)$ $=\operatorname{ht}(\tilde{M})$, it follows that $\tilde{M}$ is also complete intersection.

Thus the proof of Theorem 2.1 is complete.

3. We begin this section with the following theorem.

THEOREM 3.1. Let $k$ be a field. Let $R=k\left[\left[X_{1}, X_{2}, \ldots, X_{n}\right]\right]$ be a power series ring in $n$ variables over $k$. Let $M$ be a maximal ideal of $R[T]$. Then $M$ is complete intersection.

Proof. Let $p=R \cap M$. If $p=0$ then $\operatorname{ht}(M)=\operatorname{ht}(p)+1=1$.

Since $R[T]$ is a unique factorization domain, $M$ will be a principal ideal and hence complete intersection.

If $p \neq 0$ then let $f$ be a nonzero element of $p$. It is easy to see that there exist $Y_{1}, Y_{2}, \ldots, Y_{n} \in R$ such that $R=k\left[\left[Y_{1}, Y_{2}, \ldots, Y_{n}\right]\right]$ and $f$ as a power series in $Y_{1}, Y_{2}, \ldots, Y_{n}$ is regular in $Y_{n}$. Therefore without loss of generality we can assume that $f=f\left(X_{1}, \ldots, X_{n}\right)$ is regular in $X_{n}$. Then by the Weierstrass preparation theorem [Z-S, p. 139] there exists a unit $u\left(X_{1}, \ldots, X_{n}\right)$ in $R$ such that

$$
u\left(X_{1}, \ldots, X_{n}\right) f\left(X_{1}, \ldots, X_{n}\right)=f^{\prime}\left(X_{1}, \ldots, X_{n}\right)=X_{n}^{r}+g_{1} X_{n}^{r-1}+\cdots+g_{r}
$$

where $g_{i} \in k\left[\left[X_{1}, \ldots, X_{n-1}\right]\right]$ and $g_{i}(0,0, \ldots, 0)=0$ for $1 \leqslant i \leqslant r$. Let $S=$ $k\left[\left[X_{1}, \ldots, X_{n-1}\right]\right]\left[X_{n}\right] \subset R$. Then it also follows from the above-mentioned theorem that $S / f^{\prime} S=R / f^{\prime} R$. Therefore $S[T] / f^{\prime} S[T]=R[T] / f^{\prime} R[T]$.

Let $\tilde{M}=M \cap S[T]$. Then since $f^{\prime} \in p \cap S \subset \tilde{M}$ it follows that $\tilde{M}$ is a maximal ideal of $S[T], \quad \tilde{M} R[T]=M$ and $\mathrm{ht}(\tilde{M})=\mathrm{ht}(M)$. Since $S[T]=$ $k\left[\left[X_{1}, \ldots, X_{n-1}\right]\right]\left[X_{n}, T\right]$ by the D-G theorem $\tilde{M}$ is complete intersection. Hence $M$ is also complete intersection.

This completes the proof of Theorem 3.1.

Let $R$ be an equicharacteristic regular local ring. Let $\hat{R}$ be the completion of $R$ with respect to $\Re(R)$-adic topology. Then $\hat{R}=k\left[\left[X_{1}, \ldots, X_{n}\right]\right]$ where $k$ is the residue field of $R$ and $n=\operatorname{dim} R$.

Now we state a proposition which is a generalization of Theorem 3.1.

Proposition 3.2. Let $R$ be an equicharacteristic regular local ring. Let $\hat{R}$ be its completion with respect to $\Re(R)$-adic topology. Let $M$ be a maximal ideal of $R[T]$. Let $I=M \hat{R}[T]$. Then $\mathrm{ht}(I)=\mathrm{ht}(M)$ and $I$ is complete intersection. 
Proof. Let $\hat{R}=k\left[\left[X_{1}, \ldots, X_{n}\right]\right]$ where $k=R / \mathscr{T}(R)$. Since $M$ is locally generated by a regular sequence of length $=\mathrm{ht}(M)$ and $\hat{R}[T]$ is a faithfully flat extension of $R[T]$ it follows that $\operatorname{ht}(M)=\operatorname{ht}(I)$. If $\mathrm{ht}(M)=1$ then $M$ itself is complete intersection and therefore $I$ is also complete intersection. Now we assume that $\operatorname{ht}(M) \geqslant 2$.

Let $J=I \cap \hat{R}$. Then $\operatorname{ht}(I)=\operatorname{ht}(M) \geqslant 2$ implies that $J \neq 0$. Then as in Theorem 3.1 we can assume that $J$ contains an element $f$ such that $f \in S, S / f S=R / f R$ where $S=k\left[\left[X_{1}, \ldots, X_{n-1}\right]\right]\left[X_{n}\right]$. Moreover we can assume that $f$ is monic in $X_{n}$.

Let $I^{\prime}=I \cap S[T]$. Since $f \in I^{\prime}$ we have $\mu\left(I^{\prime} / I^{\prime 2}\right)=\mu\left(I / I^{2}\right)$ and $I^{\prime} \hat{R}[T]=I$. But $\hat{R}[T]$ is faithfully flat over $R[T], M \hat{R}[T]=I$ and $M$ is a maximal ideal of $R[T]$. Therefore $\mu\left(I / I^{2}\right)=\mu\left(M / M^{2}\right)=\mathrm{ht}(M)=\mathrm{ht}(I)$.

Since $S[T]=k\left[\left[X_{1}, \ldots, X_{n-1}\right]\right][T]\left[X_{n}\right]$ and $f \in I^{\prime}, I^{\prime}$ contains a monic polynomial in $X_{n}$ with coefficients in $k\left[\left[X_{1}, \ldots, X_{n-1}\right]\right][T]$. Since $\mu\left(I^{\prime} / I^{\prime 2}\right)=\mu\left(I / I^{2}\right)=\operatorname{ht}(I)$ $\geqslant 2$ and $\operatorname{dim} S[T] / I^{\prime}=\operatorname{dim} R[T] / I=0$ (this is easy to check) by [Mo, Theorem 5] there exists a finitely generated projective $S[T]$-module $P$ of rank $=\mu\left(I^{\prime} / I^{\prime 2}\right)$ and a surjective homomorphism $\psi: P \rightarrow I^{\prime}$. But by [L-L, Theorem 2] $P$ is free and therefore $\mu(P)=\operatorname{rank}(P)=\mu\left(I^{\prime} / I^{2}\right)$. This implies that $\mu\left(I^{\prime}\right) \leqslant \mu\left(I^{\prime} / I^{\prime 2}\right)=\mu\left(I / I^{2}\right)=$ $\operatorname{ht}(I)$. Since $I^{\prime} \hat{R}[T]=I$, we have $\mu(I) \leqslant \mu\left(I^{\prime}\right) \leqslant \operatorname{ht}(I) \leqslant \mu(I)$. Therefore $I$ is complete intersection.

This completes the proof of Proposition 3.2.

REMARK. In view of known results regarding projective modules over $R[T]$ when $R$ is regular local, one can obtain the results of $\S \S 2$ and 3 in one stroke if one can prove the following theorem.

THEOREM. Let $R$ be a regular local ring. Let $M$ be a maximal ideal of $R[T]$. Then there exists a projective $R[T]-$ module $P$ of rank $=\mathrm{ht}(M)$ and a surjective homomorphism $\psi: P \rightarrow M$.

\section{REFERENCES}

[BI] Birger Iversen, Generic local structure in commutative algebra, Lecture Notes in Math., vol. 310, Springer-Verlag, Berlin, Heidelberg and New York, 1973.

[D-G] E. D. Davis and A. V. Geramita, Generation of maximal ideals in polynomial rings, Trans. Amer. Math. Soc. 231 (1977), 497-505.

[L] H. Lindel, On a question of Bass, Quillen and Suslin concerning projective modules over polynomial rings, preprint.

[L-L] H. Lindel and W. Lütkebohmert, Projective Moduln über Polynomial Erweiterungen von Potenzreihenalgebren, Arch. Math. 28 (1977), 51-54.

[Mo] N. Mohan Kumar, On two conjectures about polynomial rings, Invent. Math. 46 (1978), 225-236.

[Mu] M. P. Murthy, Projective A[X]-modules, J. London Math. Soc. (2) 41 (1966), 453-456.

[S] J.-P. Serre, Sur les modules projectifs, Sem. Dubriel-Pisot, no. 2 (1960-61), Secrétariat Math., Paris, 1963. MR 28 \#311.

[Z-S] O. Zariski and P. Samuel, Commutative algebra. Vol. 2, Van Nostrand, Princeton, N. J., 1958.

School of Mathematics, Tata Institute of Fundamental Research, Bombay 400 005, INDia 